\title{
Avaliação hemodinâmica e metabólica da cetamina e cetamina S(+) após a reposição volêmica com hidroxietilamido 130/0,4 e solução salina hipertônica 7,5\%
}

\author{
Hemodynamic and metabolic evaluation of ketamine and $\mathrm{S}(+)$ ketamine after volemic \\ expansion with hydroxyethyl starch 130/0,4 and hypertonic saline solution
}

\author{
Nilson Oleskovicz ${ }^{\text {J Juan Carlos Duque }}{ }^{\text {II }}$ Erica Cristina Prado Guirro ${ }^{\text {III }}$ \\ Carlos Augusto Araújo Valadão ${ }^{\mathrm{IV}}$ Denise Tabacchi Fantoniv
}

RESUMO

O objetivo deste estudo foi avaliar os efeitos hemodinâmicos e metabólicos, após a administração de solução salina hipertônica (NaCL) 7,5\% ou em associação ao hidroxietilamido (HES), em cães com hipovolemia induzida e tratados com cetamina. Após a indução da hipovolemia, administrou-se $\mathrm{NaCl} 7,5 \%\left(4,0 \mathrm{ml} \mathrm{kg}{ }^{-1}\right)$ no grupo hipertônica levógira (GHL) e grupo hipertônica racêmica (GHR) ou HES 130/0,4 na mesma proporção de sangue retirado, associado a $\mathrm{NaCl}$ 7,5\% (4ml $\left.\mathrm{kg}^{-1}\right)$ no grupo hipertônica colóide levógira (GHCL) e no grupo hipertônica colóide racêmica (GHCR). Após 30 minutos, administrou-se, por via IV, cetamina levógira (CL) (5mg kg-1) no GHL e GHCL ou cetamina racêmica (CR) (10 mg kg-1) no GHR e GHCR. Empregou-se a análise de variância de uma única via com repetições múltiplas (ANOVA) $e$ o teste de Student Newman Keuls $(P \leq 0,05)$. A frequência cardíaca e a pressão arterial sistólica foram menores após a hipovolemia e após a CR. As pressões arteriais média e diastólica foram menores após a hipovolemia e cetamina. A pressão venosa central foi maior após a administração do colóide. Os índices cardíaco e sistólico foram menores após a hipovolemia em todos os grupos e, após a fase de expansão no GHL e GHR. A pressão média da artéria pulmonar foi menor após a hipovolemia em todos os grupos. A pressão de oclusão da artéria pulmonar foi maior após o colóide. $O$ índice do trabalho ventricular esquerdo foi menor após a hipovolemia no GHCL e GHCR. O índice da resistência periférica total foi maior após a hipovolemia e menor após a CL. Observou-se acidose metabólica após a hipovolemia e após a cetamina. Ocorreu acidose respiratória após a cetamina no GHL e GHR. Concluise que a administração de $\mathrm{NaCl} 7,5 \%$ associado ao HES 130/ 0,4 promove o restabelecimento imediato dos parâmetros hemodinâmicos e metabólicos no paciente hipovolêmico; a administração isolada de $\mathrm{NaCl}$ 7,5\% não é capaz de restaurar a PAM no período imediato, mas melhora os demais parâmetros hemodinâmicos e metabólicos; a administração de CR ou CL produz efeitos hemodinâmicos e metabólicos similares no paciente hipovolêmico.

Palavras-chave: solução hipertônica, hidroxietilamido, cetamina, cães, hipovolemia.

\section{ABSTRACT}

The objective of this study was to evaluate the hemodynamics and hemogasometrics effects, after the administration of hypertonic solution $(\mathrm{NaCl} 7.5 \%)$ or in association with hidroxyethyl starch 130/0.4 (HES), in dogs with induced experimental hypovolemia and treated with racemic ketamine $(R K)$ or $S(+)$ ketamine (SK). After the hypovolemia induction, administration of $\mathrm{NaCl} 7.5 \%\left(4 \mathrm{ml} \mathrm{kg}^{-1}\right)$ was performed in two groups called hypertonic $S(+)$ group (HSG) and hypertonic racemic group (HRG), or $\mathrm{NaCl} 7.5 \%$ (4 $\mathrm{ml} \mathrm{kg}^{-1}$ ) in association with HES, in the same ratio of removed blood, in two groups called hypertonic colloid $S(+)$ group (HCSG) and hypertonic colloid racemic group (HCRG). After 30 minutes, it was administered by intravenous injection, SK (5tymg kg-1) in HSG and HCSG groups, or RK $\left(10 \mathrm{mg} \mathrm{kg}^{-1}\right)$ in HRG and HCRG groups. To evaluate the significance of the results, it was used One-way Analysis of variance (ANOVA) for repeated measures and Student Newman Keuls method $(P \leq 0.05)$. The heart rate and the systolic arterial pressure were reduced after hypovolemia and administration of the RK. Mean and diastolic arterial pressure were reduced after hypovolemia and either SK or RK administration. The central venous pressure was increased after administration of the colloid. The cardiac output, index cardiac, and index systolic were reduced after hypovolemia in all groups and, after phase of expansion in HSG and HRG. The pulmonary arterial occlusion pressure was increased after colloid administration. The left ventricular work index was reduced after hypovolemia in HCSG and HCRG. The

${ }^{\mathrm{I} C e n t r o}$ de Ciências Agroveterinárias (CAV), Universidade do Estado de Santa Catarina (UDESC). Av. Luiz de Camões, 2090, 88520-000, Lages, SC, Brasil. E-mail: a2no@cav.udesc.br. Autor para correspondência.

IUniversidade de Franca (UNIFRAN), Franca, SP, Brasil.

IIIUniversidade Federal do Paraná (UFPR), Campus Palotina, Palotina, PR, Brasil.

IVUniversidade Estadual Paulista (UNESP), Jaboticabal, SP, Brasil.

${ }^{\vee}$ Univeridade de São Paulo (USP), São Paulo, SP, Brasil. 
systemic vascular resistance index was increased after hypovolemia and decreased after administration of the SK. The $\mathrm{CO}_{2}$ concentration to the end of the expiration increased after administration of ketamine in HCSG and HCRG. Metabolic acidosis was observed after hypovolemia and after administration of ketamine in all groups. Ketamine in HSG and HRG produced respiratory acidosis. It has been concluded that the administration of $\mathrm{NaCl} 7.5 \%$ by itself or in association with HES immediately restores homodynamic and metabolic parameters of the hypovolemic patient. However, the administration of $\mathrm{NaCl} 7.5 \%$ does not increase the mean arterial pressure of these patients. The administration of either SK or $R K$ produces similar homodynamic effects in hypovolemic patients.

Key words: hypertonic solution, hidroxyethyl starch, ketamine, dogs, hypovolemia.

\section{INTRODUÇÃO}

A síndrome choque caracteriza-se por hipoperfusão tissular generalizada produzida por deficiência na microcirculação, acarretando suprimento inadequado de nutrientes, como o oxigênio, levando ao metabolismo anaeróbico e à acidose metabólica, até a morte celular e falência de órgãos vitais (MILLER, 1992). A restauração da circulação melhora, transitoriamente, a condição hemodinâmica do paciente e, conseqüentemente, a perfusão tecidual. As soluções cristalóides isotônicas foram muito utilizadas, como principal fluido para reposição volêmica (VELASCO et al., 1980). No entanto, muitos estudos demonstram resultados apenas satisfatórios com o uso dessas substâncias, sobretudo no tratamento do choque hemorrágico grave. A constatação de que o emprego de pequeno volume de solução cristalóide hipertônica de cloreto de sódio ( $\mathrm{NaCl}$ 7,5\%) promovia restabelecimento imediato dos parâmetros hemodinâmicos de cães submetidos a choque hemorrágico grave fez surgir um novo conceito na terapia do choque. A infusão de $\mathrm{NaCl}$ 7,5\% aumenta o volume plasmático em duas a quatro vezes o volume infundido, aumenta a pressão arterial média (PAM), o débito cardíaco (DC) e corrige os distúrbios metabólicos, por período superior a 6 horas (VELASCO et al., 1980).

Foi comprovado que os colóides são efetivos em pequeno volume e promovem expansão volêmica de longa duração, devido à elevada pressão oncótica. Mantêm a pressão coloidosmótica intravascular, estabilizando o gradiente entre ela e a intersticial, reduzindo o extravasamento de líquidos e o possível edema intersticial (KRAMER et al., 1986). O hidroxietilamido 130/0,4 (HES 130/0,4) apresenta, em cada grupo de 10 glicoses da molécula de amido, quatro moléculas de glicoses que sofrem hidroxiacetilação especificamente no carbono dois, caracterizando-o como hidroxietilamido de peso molecular $130 \mathrm{kDa}$ e grau de substituição molar de 0,4. Quando administrado por via IV, o HES 130/0,4 possui moléculas menores (60 a $70 \mathrm{kDa}$ ) excretadas por filtração renal, enquanto que as maiores são biotransformadas pela a-amilase sérica, antes de sua excreção via filtração glomerular. Após sua infusão, ele mantém moléculas com peso entre 70 a $80 \mathrm{kDa}$, as quais permanecem acima do limiar de filtração renal durante todo o período terapêutico. Este composto apresenta uma capacidade de expansão de aproximadamente $100 \%$, ou seja, aumenta o volume plasmático na mesma proporção do volume infundido por 4 a 6 horas, o qual é comparável aos demais HES utilizados (WALTZINGER et al., 1999).

A indução anestésica em pacientes hipovolêmicos é a principal causa de mortalidade, provavelmente devido ao fato de os fármacos utilizados interferirem nos reflexos cardiovasculares e nas respostas do sistema nervoso simpático (INGWERSEN et al., 1988). A cetamina tem sido indicada para indução anestésica de pacientes em choque hemorrágico ou com hipovolemia grave, devido aos seus efeitos estimulantes cardiovasculares, produzindo aumento da freqüência cardíaca (FC), pressão venosa central (PVC), pressão da artéria pulmonar (PAP), pressão arterial média (PAM) e débito cardíaco (DC) (REIS-OLIVEIRA et al., 1980). A pressão arterial (PA) mantém-se elevada em relação à observada para barbitúricos ou anestésicos inalatórios (HASKINS \& PATZ, 1990), produzindo taxas de sobrevivência maiores àquelas observadas com halotano e pentobarbital em ratos hipovolêmicos (LONGNECKER \& STURGILL, 1976).A administração de metade da dose de cetamina levógira (CL) propiciou o retorno às atividades mais rapidamente do que nos indivíduos que receberam cetamina racêmica (CR), demonstrando que $50 \%$ da dose de CL produziu resultados clínicos equipotentes (ADAMS \& WERNER, 1997).

Neste estudo, objetivou-se avaliar a reposição volêmica, com o uso isolado de solução hipertônica $(\mathrm{NaCl} 7,5 \%)$ ou em associação ao hidroxietilamido 130/0,4, em pacientes com hipovolemia induzida, estabelecendo-se critérios comparativos entre a indução anestésica com a cetamina levógira ou cetamina racêmica, em relação aos efeitos hemodinâmico e metabólico.

\section{MATERIAL E MÉTODOS}

Foram utilizadas quatro cadelas, Beagles, castradas e esplenectomizadas, pesando entre 8 - 10kg, com 10 a 12 meses de idade. Esses animais foram 
submetidos ao protocolo experimental quatro vezes, com intervalos de 35 dias, seguindo a metodologia do quadrado latino. Nos dias de experimentação, os animais foram colocados em decúbito lateral direito, sobre colchão térmico ativo, e submetidos à indução anestésica com sevoflurano ${ }^{\mathrm{a}}$ administrado por máscara facial com fluxo diluente de oxigênio de $4 \mathrm{~L} \mathrm{~min}^{-1}$. Após intubação orotraqueal com sonda de Magill, a manutenção anestésica foi realizada com sevoflurano expirado a 2,3V\% aferida pela leitura no analisador de gases anestésicos ${ }^{\mathrm{b}}$ vaporizado com fluxo de $30 \mathrm{ml} \mathrm{kg}$ min $^{-1}$ de oxigênio a $100 \%$, administrados por meio de circuito anestésico com reinalação parcial de gases. Subseqüentemente, foi dissecada a artéria tibial dorsal para a introdução de um cateter de polietileno (22G), utilizado para mensuração da PAS e obtenção das amostras de sangue arterial. Na seqüência, introduziuse um cateter de polietileno (14G) na veia jugular direita, através do qual se introduziu o cateter de Swan Ganz . Esse último teve sua extremidade posicionada na artéria pulmonar, a qual foi confirmada pela leitura do traçado da onda de pulso no monitor do equipamento multiparamétrico ${ }^{\mathrm{d}}$.

Após o preparo, os animais foram mantidos sob anestesia geral inalatória com sevoflurano expirado a 2,3V\% durante todo o período de experimentação. Decorridos 30 minutos de estabilização da anestesia, retirou-se um volume não pré-determinado de sangue, através da artéria tibial dorsal, com taxa máxima de extração de $20 \mathrm{ml} \mathrm{min}^{-1}$, até atingir valores de PAM entre 45 e 55mmHg. A pressão foi mantida nessa faixa durante 30 minutos pela extração ou pela infusão de alíquotas adicionais de sangue. O sangue extraído foi armazenado em bolsas de transfusão sangüínea e mantido sob refrigeração, para ser reinfundido nos animais ao término de cada período de experimentação. Após a indução da hipovolemia, administraram-se injeções IV de $\mathrm{NaCl}$ a $7,5 \%$ e na dose de $4 \mathrm{ml} \mathrm{kg}^{-1}$ no GHL (grupo hipertônica cetamina levógira) e GHR (grupo hipertônica cetamina racêmica) ou solução de HES 130/ $0,4^{\mathrm{f}}$ na proporção de $1: 1$, em relação ao volume de sangue retirado de cada animal, associada a $\mathrm{NaCl} 7,5 \%$ na dose de $4 \mathrm{ml} . \mathrm{kg}^{-1}$ no GHCL (grupo hipertônica colóide cetamina levógira) e GHCR (grupo hipertônica colóide cetamina racêmica). Esse período denominado de expansão teve a duração total de 20 minutos para infusão das soluções. Após 30 minutos da fase de expansão, foi administrado, por via IV, em ensaio cego, $\mathrm{CL}^{\mathrm{g}}\left(5 \mathrm{mg} \mathrm{kg}^{-1}\right)$ no GHL $(\mathrm{n}=4)$ e GHCL $(\mathrm{n}=4)$ ou $\mathrm{CR}^{\mathrm{h}}$ $\left(10 \mathrm{mg} \mathrm{kg}^{-1}\right)$ no GHR $(\mathrm{n}=4)$ e GHCR $(\mathrm{n}=4)$. Em todos os grupos, foi administrada fluidoterapia de manutenção com solução salina fisiológica $0,9 \%$ na dose de $5 \mathrm{ml} \mathrm{kg}$ hora $^{-1}$, iniciando-se 30 minutos após a hipovolemia (T0) por até 180 minutos (T180). Como forma de evitar uma hipotensão duradoura, após a administração do agente dissociativo, administrou-se solução de Ringer com lactato de sódio, até o final do período de experimentação, em volume suficiente para estabilizar a PAM em, no mínimo, 60mmHg.

Foram mensurados e calculados os seguintes parâmetros: freqüência cardíaca (FC), pressão arterial sistólica (PAS), diastólica (PAD) e média (PAM), pressão venosa central (PVC), índice cardíaco (IC), índice sistólico (IS), pressão média da artéria pulmonar (PAPm), pressão de oclusão da artéria pulmonar (PAPo), índice trabalho ventricular esquerdo (ITVE), índice da resistência periférica total (IRPT), freqüência respiratória (f), volume corrente $(\mathrm{Vc})$, volume minuto (Vm), concentração de $\mathrm{CO}_{2}$ ao final da expiração $\left(\mathrm{ETCO}_{2}\right)$, temperatura corporal $\left(\mathrm{T}^{\circ} \mathrm{C}\right)$, hemogasometria arterial $\left(\mathrm{PaO}_{2}, \mathrm{PaCO}_{2}, \mathrm{pH}, \mathrm{Na}^{+}, \mathrm{K}^{+}, \mathrm{Ca}^{++}, \mathrm{CL}^{-}, \mathrm{HCO}_{3}{ }^{-}\right.$, $\mathrm{SaO}_{2}, \mathrm{Hb}$, Hct, déficit de base, osmolalidade, ânion gap) e glicose, nos seguintes intervalos de tempo: 30 minutos após a instrumentação (T-30), 30 minutos após a indução da hipovolemia (T0), imediatamente após a fase de expansão (T30), 15 e 30 minutos após a fase de expansão (T45 e T60), 15, 30, 45, 60, 90 e 120 minutos após a aplicação da cetamina levógira ou cetamina racêmica (T75, T90, T105, T120, T150 e T180).

Os dados coletados foram submetidos à análise de variância de uma via com repetições múltiplas (ANOVA), seguidas da comparação pelo Teste de

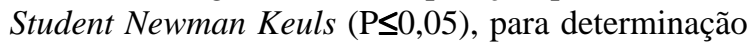
das diferenças estatísticas entre as médias ao longo do tempo dentro de cada grupo, bem como das médias em cada tempo entre os grupos. Os dados referentes ao volume de sangue retirado e o volume de Ringer Lactato administrado para manter a $\mathrm{PAM} \geq \mathrm{a} 60 \mathrm{mmHg}$ foram submetidos ao Teste $t$-pareado $(\mathrm{P} \geq 0,05)$.

\section{RESULTADOS E DISCUSSÃO}

A esplenectomia foi realizada como medida complementar para anular o efeito da espleno-contração na fase compensatória do choque, sendo que, em cães, este órgão pode contribuir, durante esta fase, liberando sangue retido em seu parênquima (DRIESSEN et al., 2001).

Após a simulação de um quadro de hemorragia aguda, e posterior estabilização do quadro clínico, através da expansão volêmica, realizada com $\mathrm{NaCl} 7,5 \%$ ou em associação com HES, os cães foram submetidos à administração de cetamina, como parte do protocolo de indução anestésica, visto que freqüentemente pacientes politraumatizados necessitam serem encaminhados para cirurgia. Nestas 
condições, muitas vezes, a hipovolemia pode ser clinicamente controlada, mas a indução anestésica torna-se obrigatória para permitir a cirurgia hemostática de urgência (HASKINS \& PATZ, 1990). Houve semelhança na resposta hemodinâmica frente à administração de CR e CL. No entanto, cabe ressaltar que a dose de CL utilizada correspondeu a $50 \%$ da dose de CR preconizada, como descrito por ADAMS \&WERNER(1997).

Observou-se redução do IC após a fase de indução da hipovolemia e aumento após a fase de expansão no GHCR e GHCL, bem como após a administração de CL no GHCL (Tabela 1). Poder-se-ia afirmar que esse acréscimo do IC, para a associação cristalóide-colóide, ocorreu pelo aumento do VS, em decorrência do maior volume plasmático circulante nesses grupos. Por outro lado, nos grupos cristalóide, o IC mantido após a fase de expansão mostra que a solução de $\mathrm{NaCl} \mathrm{7,5 \%} \mathrm{isolada} \mathrm{foi} \mathrm{capaz} \mathrm{de} \mathrm{manter} \mathrm{o} \mathrm{VS}$ e a FC.

Em relação à PAS, observou-se redução dos valores médios 15 minutos após a administração de cetamina em todos os grupos, sendo que, durante toda a fase de expansão (T30 até T60), esta variável encontrava-se próxima dos valores basais. Para a PAM, foram obtidos resultados semelhantes; no entanto, para os grupos que receberam somente solução de $\mathrm{NaCl}$ 7,5\% este parâmetro apresentou-se reduzido desde a fase de indução de hipovolemia até T180, sendo que, após a administração de cetamina, não foi observado agravamento do quadro (Tabela 1). Por outro lado, ocorreu redução da PAM, aos 15 e 30 minutos da administração de cetamina no GHCL e dos 15 até 45 minutos para o GHCR. Especula-se que a adição dos efeitos da cetamina aos da anestesia pelo sevoflurano possa ter sido a responsável pela queda da PAS e PAM em ambos os grupos, apresentando efeito mais duradouro para cetamina racêmica do que para cetamina levógira.

O ITVE foi menor de T0 até T180 no GHL, menor 15 minutos após a administração de CR, no GHR, e menor após a indução da hipovolemia no GHCL e GHCR (Tabela 1). Este fato pode ser atribuído à queda da PAM, pois, segundo VALVERDE (1991), o TVE é obtido pela relação direta da PAM x DC; no entanto, o DC e o IC mantiveram-se estáveis nestes grupos. Porém, houve redução significativa da PAM, previamente e após a administração de cetamina. Já para o GHCL, observou-se que o ITVE apresentou resultado maior após a administração de cetamina; porém, neste caso, este aumento é perfeitamente justificável, pois, embora a PAM tenha sido menor, o DC aumentou significativamente neste grupo após a administração de cetamina.
Ocorreu aumento da $\mathrm{PaCO}_{2}$ no GHL e GHR, imediatamente após a administração de cetamina. Nesse sentido, embora não tenha sido evidenciada diferença estatística entre o Vc e Vm, os valores observados foram menores nesses grupos, ou seja, poder-se-ia afirmar que, embora a cetamina não tenha reduzido a f, houve tendência de redução da amplitude respiratória, fazendo com que ocorresse um acúmulo de $\mathrm{CO}_{2}$, nesses grupos (Tabela 1). Da mesma forma, observou-se uma diminuição do pH, após a administração de cetamina, no GHL, GHR e GHCR. Corroboram estes dados os relatos de HASKINS \& PATZ (1990), que descrevem que a CR aumenta a FC, PAM, PAPm e a $\mathrm{PaCO}_{2}$, além de diminuir significativamente o $\mathrm{Vm}, \mathrm{PaO}_{2}$ e o $\mathrm{pH}$, produzindo depressão respiratória transitória em cães hipovolêmicos. Segundo VALADÃO (2002), a CR é um depressor respiratório moderado, mas em geral não afeta os gases sangüíneos. Entretanto, em alguns pacientes, pode produzir marcante hipóxia e hipercapnia, especialmente quando usada em associação com fármacos depressores do SNC.

$\mathrm{O} \mathrm{ETCO}_{2}$ aumentou no GHCL e GHCR, imediatamente após a administração de cetamina (T75), no entanto não houve alteração da $\mathrm{PaO}_{2}$ e da $\mathrm{PaCO}_{2}$. Os valores do Vc e Vm foram maiores após a administração de cetamina, embora não tenham sido observadas diferenças significativas entre os tempos. Embora a f tenha apresentado uma forte tendência de reduzir-se, a amplitude respiratória foi maior, e a relação ventilação/perfusão melhorou devido, provavelmente, ao maior volume circulante e à ação mais prolongada do colóide em manter a perfusão periférica, justificando os valores de $\mathrm{PaCO}_{2}$ normais e a maior eliminação do $\mathrm{CO}_{2}$, representado pelo aumento do $\mathrm{ETCO}_{2}$ nestes grupos.

Houve aumento da f após a indução da hipovolemia no GHR e GHCR. Segundo JOHNSON et al. (1985), o aumento do tônus no sistema respiratório resulta da estimulação periférica dos receptores pulmonares e dos quimiorreceptores carotídeos e da hipoperfusão do centro medular da respiração. A resposta inicial às alterações nos gases sangüíneos visa a incrementar o $\mathrm{Vm}$, por meio de taquipnéia. No entanto, muitas vezes, esta resposta é incapaz de suprir as necessidades ventilatórias, pois o aumento do Vm e a diminuição do DC incrementam o desequilíbrio ventilação/perfusão (HASKINS \& PATZ, 1990).

Em relação à hemoglobina e ao hematócrito, foram observadas reduções dos valores no GHCL e GHCR. Estes resultados corroboram os descritos por SANDER et al. (2003), que observaram decréscimo da $\mathrm{Hb}$, Hct e plaquetas, quando infundiram HES 130/0,4 durante 6 horas. Este fato deve-se provavelmente à 
Tabela 1 - Médias e respectivos desvios-padrão dos efeitos da administração da cetamina racêmica (CR) ou cetamina levógira (CL) na PAM ( $\mathrm{mmHg}$ ), IC $\left(\mathrm{L} \mathrm{min}{ }^{-1} \mathrm{~m}^{-2}\right)$, ITVE $\left(\mathrm{kg} \mathrm{x} \mathrm{m} \cdot \mathrm{min}^{-1} \mathrm{x} \mathrm{m} \mathrm{m}^{2}\right)$ e $\mathrm{PaCO}_{2}(\mathrm{mmHg})$ de cadelas hipovolêmicas pré-tratadas com $\mathrm{NaCl} 7,5 \%$ associada ou não ao HES (130/0,4).

\begin{tabular}{|c|c|c|c|c|c|c|c|c|c|c|c|}
\hline & $\mathrm{T}-30$ & T0 & Т30 & T45 & T60 & T75 & T90 & T105 & $\mathrm{T} 120$ & $\mathrm{~T} 150$ & T180 \\
\hline & - & & & ----- & são arte & média ( & Hg)----- & ------- & --------. & ------ & \\
\hline \multirow[t]{2}{*}{ GHL } & 100 & $51^{*}$ & $80 *$ & $82 *$ & $67 *$ & $57 *$ & $67 *$ & $72 *$ & $69 *$ & 73* & $70 *$ \\
\hline & \pm 6 & \pm 1 & \pm 8 & \pm 4 & \pm 4 & \pm 3 & \pm 3 & \pm 2 & \pm 3 & \pm 6 & \pm 4 \\
\hline \multirow[t]{2}{*}{ GHR } & 86 & $53 *$ & 73* & $68 *$ & $60 *$ & $43 *$ & $62 *$ & $62 *$ & $66^{*}$ & $68^{*}$ & 76 \\
\hline & \pm 6 & \pm 3 & \pm 7 & \pm 8 & \pm 6 & \pm 2 & \pm 3 & \pm 2 & \pm 5 & \pm 6 & \pm 3 \\
\hline \multirow[t]{2}{*}{ GHCL } & 92 & $54^{*}$ & 74 & 79 & 76 & $66^{*}$ & $60 *$ & 71 & 76 & 80 & 80 \\
\hline & \pm 6 & \pm 2 & \pm 3 & \pm 7 & \pm 8 & \pm 9 & \pm 6 & \pm 4 & \pm 5 & \pm 4 & \pm 4 \\
\hline \multirow[t]{3}{*}{ GHCR } & 87 & $55^{*}$ & 74 & 76 & 71 & $52 *$ & $60 *$ & $62 *$ & 71 & 77 & 70 \\
\hline & \pm 4 & \pm 4 & \pm 8 & \pm 7 & \pm 6 & \pm 6 & \pm 6 & \pm 5 & \pm 4 & \pm 5 & \pm 10 \\
\hline & & & & 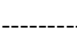 & dice ca & $\operatorname{co}(\mathrm{L} / \mathrm{m}$ & $\left.7^{2}\right)---\cdot$ & & & & \\
\hline \multirow[t]{2}{*}{ GHL } & 4,7 & $2,4^{*}$ & 3,8 & 3,8 & $3,4^{*}$ & 3,7 & 4,3 & 4,4 & 4,1 & 3,6 & 3,8 \\
\hline & $\pm 0,4$ & $\pm 0,2$ & $\pm 0,3$ & $\pm 0,3$ & $\pm 0,2$ & $\pm 0,3$ & $\pm 0,1$ & $\pm 0,2$ & $\pm 0,2$ & $\pm 0,3$ & $\pm 0,4$ \\
\hline \multirow[t]{2}{*}{ GHR } & 3,7 & $2,1^{*}$ & 3,2 & 3,2 & 3,7 & 3,7 & 4,4 & 4,2 & 5,0 & 4,7 & 4,1 \\
\hline & $\pm 0,6$ & $\pm 0,2$ & $\pm 0,2$ & $\pm 0,2$ & $\pm 0,4$ & $\pm 0,3$ & $\pm 0,1$ & $\pm 0,1$ & $\pm 0,3$ & $\pm 0,9$ & $\pm 0,7$ \\
\hline \multirow[t]{2}{*}{ GHCL } & 4,9 & $2,0 *$ & $4,4 \#$ & $5,5 \#$ & 5,4 & $5,6 \#$ & 4,9 & 5,0 & 4,8 & 5,2 & 5,1 \\
\hline & $\pm 0,4$ & $\pm 0,1$ & $\pm 0,3$ & $\pm 0,2$ & $\pm 0,3$ & $\pm 0,5$ & $\pm 0,3$ & $\pm 0,4$ & $\pm 0,5$ & $\pm 0,5$ & $\pm 0,3$ \\
\hline \multirow[t]{3}{*}{ GHCR } & 4,4 & $2,4^{*}$ & $5,1 \#$ & $5,0 \#$ & 5,0 & 4,0 & 4,4 & 4,3 & 4,4 & 4,3 & 3,6 \\
\hline & $\pm 0,5$ & $\pm 0,2$ & $\pm 0,3$ & $\pm 0,3$ & $\pm 0,3$ & $\pm 0,3$ & $\pm 0,5$ & $\pm 0,5$ & $\pm 0,6$ & $\pm 0,6$ & $\pm 0,5$ \\
\hline & & & - & ce do $t$ & ho vent & lar esqu & $(\mathrm{kg} \times \mathrm{n}$ & $\mathrm{n}^{-1} \times \mathrm{m}^{2}$ & & & \\
\hline \multirow[t]{2}{*}{ GHL } & 6,1 & $1,6^{*}$ & $3,8^{*}$ & $4,0 *$ & $2,9 *$ & $2,7^{*}$ & $3,7^{*}$ & $4,0 *$ & $3,7^{*}$ & $3,5^{*}$ & $3,3 *$ \\
\hline & $\pm 0,7$ & $\pm 0,1$ & $\pm 0,3$ & $\pm 0,1$ & $\pm 0,1$ & $\pm 0,3$ & $\pm 0,1$ & $\pm 0,2$ & $\pm 0,2$ & $\pm 0,5$ & $\pm 0,3$ \\
\hline \multirow[t]{2}{*}{ GHR } & 4,2 & $1,3^{*}$ & 3,1 & 2,7 & 2,8 & $2,0 *$ & 3,5 & 3,3 & 4,1 & 4,5 & 4,0 \\
\hline & $\pm 0,7$ & $\pm 0,1$ & $\pm 0,2$ & $\pm 0,2$ & $\pm 0,3$ & $\pm 0,2$ & $\pm 0,2$ & $\pm 0,1$ & $\pm 0,5$ & $\pm 0,6$ & $\pm 0,7$ \\
\hline \multirow[t]{2}{*}{ GHCL } & 5,9 & $1,4^{*}$ & 4,2 & $5,8 \#$ & 5,5\# & 4,9\# & 3,8 & $4,6 \#$ & 4,8 & 5,6 & 4,8 \\
\hline & $\pm 0,9$ & $\pm 0,1$ & $\pm 0,3$ & $\pm 0,6$ & $\pm 0,8$ & $\pm 0,8$ & $\pm 0,3$ & $\pm 0,4$ & $\pm 0,5$ & $\pm 0,7$ & $\pm 0,8$ \\
\hline \multirow[t]{3}{*}{ GHCR } & 5,1 & $2,0 * \#$ & 5,0 & $5,0 \#$ & $4,6 \#$ & 2,8 & 3,4 & 3,5 & 4,0 & 4,3 & 3,2 \\
\hline & $\pm 0,7$ & $\pm 0,2$ & $\pm 0,8$ & $\pm 0,7$ & $\pm 0,4$ & $\pm 0,5$ & $\pm 0,5$ & $\pm 0,4$ & $\pm 0,5$ & $\pm 0,6$ & $\pm 0,6$ \\
\hline & & & & & --------1 & $\mathrm{O}_{2}(\mathrm{mml}$ & & & & & \\
\hline \multirow[t]{2}{*}{ GHL } & 40,5 & 36,2 & 39,2 & 38,0 & 36,4 & $51,9 *$ & 42,7 & 38,0 & 37,0 & 35,1 & 36,7 \\
\hline & $\pm 0,7$ & $\pm 2,6$ & $\pm 1,5$ & $\pm 3,0$ & $\pm 3,2$ & $\pm 5,9$ & $\pm 3,6$ & $\pm 3,2$ & $\pm 2,3$ & $\pm 2,4$ & $\pm 2,5$ \\
\hline \multirow[t]{2}{*}{ GHR } & 40,7 & 35,8 & 39,7 & 38,1 & 32,5 & $51,0 *$ & 41,2 & 38,6 & 37,0 & 33,9 & 35,3 \\
\hline & $\pm 3,8$ & $\pm 2,4$ & $\pm 2,9$ & $\pm 2,9$ & $\pm 4,2$ & $\pm 3,7$ & $\pm 3,0$ & $\pm 1,7$ & $\pm 2,8$ & $\pm 1,3$ & $\pm 1,4$ \\
\hline \multirow[t]{2}{*}{ GHCL } & 41,7 & 35,0 & 39,5 & 32,3 & 30,0 & 41,3 & 35,4 & 41,5 & 36,8 & 38,2 & 37,9 \\
\hline & $\pm 2,6$ & $\pm 5,4$ & $\pm 3,4$ & $\pm 5,2$ & $\pm 8,5$ & $\pm 10,0$ & $\pm 10,5$ & $\pm 2,0$ & $\pm 5,8$ & $\pm 2,9$ & $\pm 3,1$ \\
\hline \multirow[t]{2}{*}{ GHCR } & 39,5 & 32,1 & 38,4 & 40,1 & 36,4 & 46,0 & 41,3 & 38,3 & 37,4 & 34,6 & 34,5 \\
\hline & $\pm 1,5$ & $\pm 3,5$ & $\pm 2,9$ & $\pm 3,8$ & $\pm 4,5$ & $\pm 11,5$ & $\pm 6,8$ & $\pm 5,3$ & $\pm 7,8$ & $\pm 5,7$ & $\pm 4,4$ \\
\hline
\end{tabular}

* Significativamente diferente de T-30; \# Significativamente diferente dos demais grupos.

hemodiluição acarretada pela infusão do colóide. No GHL e GHR, também foi observado redução da Hb após a fase de expansão. E, de acordo com KOLSENPETERSEN (2004), a infusão de solução salina hipertônica promove hemodiluição justificando a redução nestas variáveis.

Referente aos valores de $\mathrm{Na}^{+}$, foram observados aumentos em todos os grupos, após a administração de $\mathrm{NaCl}$ 7,5\%. Estes resultados eram esperados e foram semelhantes aos descritos por KRAMER et al. (1986). Os resultados referentes ao DB encontrados neste estudo estão de acordo com os descritos por HASKINS et al. (2005), que observaram que o DB aumentou de -2,2 para -6,3mEq L ${ }^{-1}$. Da mesma forma, os resultados de DB, glicose e eletrólitos foram semelhantes aos obtidos por GUNDERSEN et al. (2001), que observaram alterações abruptas, em ratos submetidos a choque hemorrágico, sendo que os valores basais de DB de $0,3 \pm 0,2 \mathrm{mEq} \mathrm{L} \mathrm{L}^{-1}$ aumentaram para $11,2 \pm 1,0 \mathrm{mEq} \mathrm{L} \mathrm{L}^{-1}, 15$ minutos após a indução da hemorragia. Além disso, os mesmos autores da glicose sérica durante todo o período de avaliação; entretanto, não observaram diferenças nas concentrações de $\mathrm{Na}^{+}$, $\mathrm{K}^{+}$e $\mathrm{Ca}^{++}$após a indução do choque hemorrágico. 
A administração de $\mathrm{NaCl}$ 7,5\% não restaurou a PAM, que havia sido reduzida pela hipovolemia durante e após a fase de expansão e, tampouco, a injeção dos agentes dissociativos recuperou ou produziu diferenças nas PA entre os grupos. Por outro lado, a associação $\mathrm{NaCl}$ 7,5\% com HES 130/0,4 manteve estável a PAM durante e após a fase de expansão. Estes dados estão de acordo com os descritos por SANDER et al. (2003) em pacientes hipovolêmicos tratados com HES 130/0,4 ou HES 200/0,5. Assim, notou-se que a reposição volêmica com solução de $\mathrm{NaCl} 7,5 \%$ ou em associação com hidroxietilamindo 130/ 0,4 apresenta algumas diferenças importantes. Dessa maneira, foi observada maior estabilidade hemodinâmica com o emprego do hidroxietilamindo 130/0,4, fato esse que se justifica pelos valores maiores de IC, IS, PVC, PAPo e ITVE após a fase de expansão, no grupo GHCL e GHCR, em comparação aos demais grupos. Esses resultados corroboram os descritos por KREIMEIER et al. (1993), que descreveram que a $\mathrm{NaCl}$ 7,5\% apresenta efeitos circulatórios de curta duração, devido ao aumento na osmolalidade plasmática e rápido equilíbrio entre os compartimentos extracelular e intracelular. Dessa forma, acredita-se que a associação de colóides produz efeitos mais consistentes pelo aumento da osmolalidade plasmática, o que resulta em maior mobilização de água intracelular e, conseqüentemente, no aumento da pressão oncótica plasmática.

A quantidade de solução de Ringer com lactato de sódio administrado para manter a PAM $\geq 60 \mathrm{mmHg}$ foi significativamente maior no GHR $(437,50 \pm 51,53 \mathrm{ml})$, quando comparado ao GHL $(100,00 \pm 70,71 \mathrm{ml})$, GHCL $(175,00 \pm 112,73 \mathrm{ml})$ e GHCR $(137,50 \pm 137,50 \mathrm{ml})$. Nesse sentido, observou-se que, nos animais do GHR, administraram somente $40 \mathrm{ml} \mathrm{de} \mathrm{NaCl}$ 7,5\% para uma perda média de $228 \mathrm{ml}$ de sangue. Este fato explica, em parte, a maior necessidade de solução cristalóide administrada nesse grupo após a administração da CR para manter a PAM estabilizada em60mmHg.

\section{CONCLUSÃO}

Conclui-se que a administração de HES 130/ 0,4 associado a $\mathrm{NaCl} 7,5 \%$ promove o restabelecimento imediato dos parâmetros hemodinâmicos e metabólicos no paciente hipovolêmico. Além disso, a administração isolada de solução salina hipertônica não é capaz de restaurar a PAM no período imediato, mas melhora os demais parâmetros hemodinâmicos e metabólicos. Por fim, a administração de CR ou CL produz efeitos hemodinâmicos e metabólicos similares no paciente hipovolêmico.

\section{AGRADECIMENTOS}

À Fundação de Amparo à Pesquisa do Estado de São Paulo (FAPESP), pela concessão de bolsa de estudos e pelo auxílio financeiro, processos 03/01111-9 e 03/13671-9.

\section{COMITÊ DE ÉTICA E BIOSSEGURANÇA}

Este estudo foi aprovado pela Comissão de Ética e do Bem-Estar dos Animais (CEBEA) do Campus de Jaboticabal da UNESP, conforme protocolo $\mathrm{n}^{\circ} 005750$.

\section{FONTES DE AQUISIÇÃO}

aSevocris, Cristália Produtos Químicos e Farmacêuticos Ltda, Itapira, SP.

'Ohmeda - Mod. Excel 210 SE - processo FAPESP 97/106684.

'Cateter Swan Ganz Ped. - 75 cm/5F (4 vias), 132F5, Edwards Lifesciences, São Paulo, SP.

dDixtal DX2010, Mod. PAI, Manaus, AM, Brasil. - Proc. FAPESP 96/02877-0.

eSolução hipertônica Cloreto de Sódio a 7,5 \%, Áster Produtos Médicos Ltda, Sorocaba, SP.

${ }^{f}$ Voluven 6\%, $(130 / 0,4)$ Fresenius-Kabi, Campinas, SP.

${ }^{g}$ Ketamin $\mathrm{S}(+), 50 \mathrm{mg} \cdot \mathrm{ml}^{-1}$, Cristália Produtos Químicos e Farmacêuticos Ltda, Itapira, SP.

${ }^{h} K e t a m i n, 50 \mathrm{mg} \cdot \mathrm{ml}^{-1}$, Cristália Produtos Químicos e Farmacêuticos Ltda, Itapira, SP.

\section{REFERÊNCIAS}

ADAMS, H.A.; WERNER, C. (S)- ketamine reinaisssance of a substance? Anaesthesist, v.46, n.12, p.1026-1042, 1997.

DRIESSEN, B. et al. Inadequancy of low-volume resusciation with hemoglobin-based oxygen carrier hemoglobin glutamer200 (bovine) in canine hipovolemia. Journal Veterinary Pharmacologic Therapy, v.24, p.61-71, 2001.

GUNDERSEN, Y. et al. Moderate hypothermia blunts the inflamatory response and reduces organ injury after acute haemorrhage. Acta Anesthesiologica Scandinavica, v.45, p.994-1001, 2001.

HASKINS, S.C.; PATZ, J.D. Ketamine in hypovolemic dogs. Critical Care Medicine, v.18, p.625-629, 1990.

HASKINS, S.C. et al. The effect of moderate hipovolemia on cardiopulmonary function in dogs. Journal Veterinary Emergency and Critical Care, v.15, p.100-109, 2005.

INGWERSEN, W. et al. Cardiopulmonary effects of a ketamine/ acepromazine combination in hypovolemic cats. Canadian Journal Veterinary Research, v.52, p.423-427, 1988.

JOHNSON, G. et al. Morphological differences in cutaneous and skeletal muscle vasculature during compensatory and decompensatory hemorrhagic hypotension. Circulatory Shock, v.15, p.111, 1985. 
KOLSEN-PETERSEN, J.A. Imune effect of hypertonic saline: fact or fiction? Acta Anesthesiologica Scandinavica, v.48, p.667-678, 2004

KRAMER, G.C. et al. Small volume ressuscitation with hypertonic saline dextram solution. Surgery, v.100, p.239246, 1986.

KREIMEIER, U. et al. Small-volume hyperosmolar resuscitation. Acta Anaesthesiologica Scandinavica, v.41, p.302-306, 1993

LONGNECKER, D.E.; STURGILL, B.C. Influence of anesthetic agent on survival following hemorrhage. Anesthesiology, v.45, p.516-521, 1976.

MILLER, R.D. Anestesia para cirurgia nos traumatismos. In GIESECKE, A.N.; LAWRENCE, D.E. Livro texto de anestesiologia. 2.ed. Philadelphia: Saunders, 1992. V.2, p.1877-1893

REIS-OLIVEIRA, M.P. et al. Efeitos cardiovasculares da quetamina em cães hipovolêmicos. Revista Brasileira de Anestesiologia, v.30, p.167-172, 1980.
SANDER, O. et al. Equivalence of hydroxyethyl starch HES 130/0,4 and HES 200/0,5 for perioperative volume replacent in major gynecologyco surgery. Acta Anaesthesiologica Scandinavica, v.47, p.1151-1158, 2003.

VALADÃO, C.A.A. Anestésicos dissociativos. In: FANTONI, D.T.; COTOPASSI, S.R. Anestesia em cães e gatos. São Paulo: Roca, 2002. p.165-173.

VALVERDE, A. Comparison of the hemodynamic effects of halothane alone and halothane combined with epidurally adminstred morphine for anesthesia in ventilated dogs. American Journal Veterinary Research, v.52, n.3, p.505509, 1991

VELASCO, I.T. et al. Hypertonic $\mathrm{NaCl}$ and severe hemorrhagic shock. American Journal Physiology, v.239, p.664-673, 1980 .

WALTZINGER J. et al. Effect of new HES specification (6\% HES 130/0,4) on blood and plasma volumes after bleeding in 12 healthy male volunteers. Clinical Drug Investigation, v.17, p.119-125, 1999. 